\title{
FIVE PLANETS ORBITING 55 CANCRI $^{1}$
}

\author{
Debra A. Fischer, ${ }^{2}$ Geoffrey W. Marcy, ${ }^{3}$ R. Paul Butler, ${ }^{4}$ Steven S. Vogt, ${ }^{5}$ Greg Laughlin, ${ }^{5}$ \\ Gregory W. Henry, ${ }^{6}$ David Abouav, ${ }^{2}$ Kathryn M. G. Peek, ${ }^{3}$ Jason T. Wright, ${ }^{3}$ \\ John A. Johnson, ${ }^{3}$ Chris McCarthy, ${ }^{2}$ and Howard IsaAcson ${ }^{2}$ \\ Received 2007 May 8; accepted 2007 October 24
}

\begin{abstract}
We report 18 years of Doppler shift measurements of a nearby star, 55 Cancri, that exhibits strong evidence for five orbiting planets. The four previously reported planets are strongly confirmed here. A fifth planet is presented, with an apparent orbital period of 260 days, placing it $0.78 \mathrm{AU}$ from the star in the large empty zone between two other planets. The velocity wobble amplitude of $4.9 \mathrm{~m} \mathrm{~s}^{-1}$ implies a minimum planet mass $M \sin i=45.7 M_{\oplus}$. The orbital eccentricity is consistent with a circular orbit, but modest eccentricity solutions give similar $\chi_{\nu}^{2}$ fits. All five planets reside in low-eccentricity orbits, four having eccentricities under 0.1. The outermost planet orbits 5.8 AU from the star and has a minimum mass $M \sin i=3.8 M_{\mathrm{Jup}}$, making it more massive than the inner four planets combined. Its orbital distance is the largest for an exoplanet with a well-defined orbit. The innermost planet has a semimajor axis of only $0.038 \mathrm{AU}$ and has a minimum mass, $M \sin i$, of only $10.8 M_{\oplus}$, making it one of the lowest mass exoplanets known. The five known planets within 6 AU define a minimum-mass protoplanetary nebula to compare with the classical minimum-mass solar nebula. Numerical $N$-body simulations show this system of five planets to be dynamically stable and show that the planets with periods of 14.65 and 44.3 days are not in a mean motion resonance. Millimagnitude photometry during 11 years reveals no brightness variations at any of the radial velocity periods, providing support for their interpretation as planetary.
\end{abstract}

Subject headings: planetary systems — stars: individual (55 Cancri, HD 75732, $\rho^{1}$ Cancri)

Online material: machine-readable table

\section{INTRODUCTION}

One of the first few detected exoplanets was a planetary companion to 55 Cancri (Butler et al. 1997). At the time, eight years of Doppler measurements from Lick Observatory revealed a 14.6 day wobble in $55 \mathrm{Cnc}$ as it was gravitationally perturbed by a Jupiter-mass planet. Superimposed on this 14.6 day Doppler periodicity was an additional trend showing clear curvature and indicating that $55 \mathrm{Cnc}$ was host to a second orbiting body, likely of planetary mass.

Additional Doppler measurements through 2002 uncovered the full Doppler cycle with a period of $\sim 14 \mathrm{yr}$, caused by a planet with a minimum mass $M \sin i=4 M_{\text {Jup }}$ orbiting $\sim 5.5 \mathrm{AU}$ from $55 \mathrm{Cnc}$ (Marcy et al. 2002). This was the first giant planet found with an orbital radius similar to the giant planets in our solar system. A third Doppler periodicity of 44.3 days was also apparent in those data, indicating a third Jupiter-mass planet in the system, this one orbiting 0.25 AU from the star (Marcy et al. 2002). This was the second planetary system found to have three planets, the first being that around $v$ Andromedae (Butler et al. 1999).

By combining these velocities from Lick Observatory with over 100 precise Doppler measurements obtained in one year at

\footnotetext{
1 Based on observations obtained at the W. M. Keck Observatory, which is operated jointly by the University of California and the California Institute of Technology. Keck time has been granted by both NASA and the University of California.

2 Department of Physics and Astronomy, San Francisco State University, San Francisco, CA 94132; fischer@stars.sfsu.edu.

3 Department of Astronomy, University of California, Berkeley, CA 947203411 .

4 Department of Terrestrial Magnetism, Carnegie Institution of Washington, 5241 Broad Branch Road NW, Washington, DC 20015-1305.

5 UCO/Lick Observatory, University of California, Santa Cruz, CA 95064.

6 Center of Excellence in Information Systems, Tennessee State University, 3500 John A. Merritt Boulevard, Box 9501, Nashville, TN 37209.
}

the Hobby-Eberly Telescope, along with measurements from the Elodie spectrometer at Haute Provence, McArthur et al. (2004) identified a fourth planet having a small minimum mass of $M \sin i=14 M_{\oplus}$ with an orbital period of 2.8 days. This planet was one of the first three Neptune-mass planets discovered, along with the planets orbiting GJ 436 (Butler et al. 2004; Maness et al. 2007) and $\mu$ Arae (Santos et al. 2004). The detection of this Neptune-mass planet made $55 \mathrm{Cnc}$ the first system known to contain four planets. McArthur et al. (2004) also used the Fine Guidance Sensor on the Hubble Space Telescope (HST) to carry out astrometry of $55 \mathrm{Cnc}$ and estimated the inclination of the orbital plane of the outer planet to be $i=53^{\circ} \pm 6.8^{\circ}$.

This four-planet system left a large, dynamically empty gap between 0.25 and $6 \mathrm{AU}$. Numerical simulations suggested that hypothetical planets in this gap would be dynamically stable, including the interesting possibility of terrestrial-mass planets in the habitable zone between 0.5 and 2 AU (Marcy et al. 2002; Raymond et al. 2006).

In 2004, we noticed modest peaks in the periodogram at 260 and 470 days, indicating possible planets at those periods, which motivated our continued intense Doppler observations. Wisdom (2005) carried out an independent analysis of the combined published Doppler measurements and identified a 260 day periodicity, implying a new planet with a minimum mass of $1.8 M_{\mathrm{Nep}}=$ $31 M_{\oplus}$. It is not uncommon for modest peaks in the periodogram to fluctuate in their confidence level with the addition of new data, especially for cases where the radial velocity amplitudes are comparable to the precision of Doppler measurements, so we intensified our observations of this star. Here we add 115 additional radial velocity measurements from Lick Observatory and 70 radial velocity measurements from Keck Observatory to our previous data set and find that the false-alarm probability for a 260 day signal has strengthened and is present in data sets from both observatories independently. 
A stellar companion orbits $55 \mathrm{Cnc}$ as well. It is a 13th magnitude M dwarf located roughly $1000 \mathrm{AU}$ away, certainly bound to $55 \mathrm{Cnc} \mathrm{A}$ as the radial velocities are nearly the same. The occurrence, dynamics, and final properties of planetary systems may well be affected by such stellar companions, as indicated in observational studies by Eggenberger et al. (2004) and Raghavan et al. (2006). Thus, 55 Cnc offers a test of the effects of binary companions on the architecture of complex planetary systems.

Spitzer Space Telescope results for $55 \mathrm{Cnc}$ by Bryden et al. (2006) show that the observed 24 and $70 \mu$ m flux densities are comparable to the predicted brightness of the stellar photosphere, indicating no infrared excess above the errors. The corresponding upper limit to the fractional infrared luminosity is $8 \times 10^{-6}$, or about 80 zodis. A detectable scattered light disk was also ruled out by its nondetection in HST NICMOS data by Schneider et al. (2001).

Here we provide Doppler measurements from both Lick and Keck that significantly augment the 2002 set from Lick Observatory alone. These measurements span a longer time baseline and contain higher Doppler precision with the addition of new Keck velocities, offering a chance to reassess all of the planets around $55 \mathrm{Cnc}$.

\section{PROPERTIES OF 55 CANCRI}

The star 55 Cnc $\left(=\right.$ HD $75732=\rho^{1}$ Cnc A $=$ HR $3522=$ HIP 43587) has an apparent brightness $V=5.96$ and Hipparcos parallax of $79.8 \pm 0.84$ mas, ${ }^{7}$ implying a distance of $12.5 \pm$ $0.13 \mathrm{pc}$ and absolute visual magnitude $M_{V}=5.47$. Using spectra from the California and Carnegie Planet Search, Valenti \& Fischer (2005) derived $T_{\text {eff }}=5234 \pm 30 \mathrm{~K}, \log g=4.45 \pm 0.08$, $v \sin i=2.4 \pm 0.5 \mathrm{~km} \mathrm{~s}^{-1}$, and $[\mathrm{Fe} / \mathrm{H}]=+0.31 \pm 0.04$. Indeed, $55 \mathrm{Cnc}$ is so metal-rich as to be in the fifth metallicity percentile of stars within 25 pc (Valenti \& Fischer 2005). Using a bolometric correction that accounts for the high metallicity (VandenBerg \& Clem 2003), we calculate a stellar luminosity of $0.6 L_{\odot}$. The effective temperature, spectroscopic surface gravity, and intrinsic luminosity are all consistent with a spectral classification of this star as K0/G8 V. The star is chromospherically inactive, with a Mt. Wilson $S$-value of 0.22 (averaged during the past seven years of our measurements) implying $\log R_{\mathrm{HK}}^{\prime}=$ -4.84 , indicating a modest age of $2-8 \mathrm{Gyr}$, where $2 \mathrm{Gyr}$ is a strong lower limit on age. The rotation period, calibrated to this chromospheric activity, is estimated to be 39 days (Noyes et al. 1984; Wright et al. 2004). However, for metal-rich stars, chromospheric emission at the $\mathrm{Ca}$ II $\mathrm{H}$ and $\mathrm{K}$ lines remains poorly calibrated as a diagnostic of rotation and age. A more complete discussion of the chromospheric activity and implied stellar properties is given by Marcy et al. (2002).

The mass of $55 \mathrm{Cnc}$ is best determined by associating its measured effective temperature, luminosity, and metallicity with models of stellar interiors. Using the well known Yale models, (Yi et al. 2004), Valenti \& Fischer (2005) found a stellar mass of $0.92 \pm 0.05 M_{\odot}$. Takeda et al. (2007) have also derived modified stellar evolutionary models using the Yale Stellar Evolution Code to match the observed spectroscopic parameters from Valenti \& Fischer (2005). They derive a stellar mass for $55 \mathrm{Cnc}$ of $0.96 \pm$ $0.05 M_{\odot}$ with the uncertainty corresponding to the $99.7 \%$ credibility intervals of the Bayesian posterior probability distributions. Here we simply adopt the average of these two estimates, giving $M=0.94 \pm 0.05 M_{\odot}$ for the mass of $55 \mathrm{Cnc}$. We note that the adopted uncertainty in stellar mass implies fractional errors in

\footnotetext{
${ }^{7}$ Vizier Online Data Catalog, 1239 (ESA, 1997).
}

TABLE 1

Velocities for 55 CANCRi: Lick and Keck

\begin{tabular}{|c|c|c|c|}
\hline $\mathrm{JD}-2,440,000$ & $\begin{array}{l}\text { Radial Velocity } \\
\qquad\left(\mathrm{m} \mathrm{s}^{-1}\right)\end{array}$ & $\begin{array}{l}\text { Uncertainty } \\
\left(\mathrm{m} \mathrm{s}^{-1}\right)\end{array}$ & Telescope \\
\hline $7578.730 \ldots \ldots \ldots \ldots \ldots \ldots$ & 25.67 & 9.7 & Lick \\
\hline $7847.044 \ldots \ldots$ & 3.91 & 8.4 & Lick \\
\hline 8017.688 & 31.45 & 7.5 & Lick \\
\hline 8375.669 & -31.38 & 8.8 & Lick \\
\hline
\end{tabular}

NoтE.-Table 1 is published in its entirety in the electronic edition of the Astrophysical Journal. A portion is shown here for guidance regarding its form and content.

the derived planetary masses of $8 \%$ in addition to errors in the orbital parameters.

\section{DOPPLER-SHIFT MEASUREMENTS}

We have obtained 636 observations of the G8 main-sequence star $55 \mathrm{Cnc}$ over the past 18 years. We generally obtain two or three consecutive observations and bin them to increase the velocity precision and accuracy. Here we present 250 binned velocity measurements made at Lick Observatory from 1989 to 2007 , and 70 binned velocity measurements made at the Keck Observatory from 2002 to 2007 . The Lick spectra were obtained using both the $3 \mathrm{~m}$ telescope and the Coudé Auxiliary Telescope, which both feed the Hamilton optical echelle spectrometer (Vogt 1987). A detailed description of the setup of the Hamilton spectrometer, its calibrating iodine absorption cell, and the method of extracting Doppler measurements for $55 \mathrm{Cnc}$ are given in Butler et al. (1996) and in Marcy et al. (2002). The Keck spectra were obtained with HIRES (High Resolution Echelle Spectrometer; Vogt et al. 1994), and a description of that setup and Doppler measurements are given in Butler et al. (2006).

At both telescopes, we place a cylindrical, Pyrex cell filled with molecular iodine gas in the light path of the telescope, just before the spectrometer slit, to superimpose sharp absorption lines of known wavelength on the stellar spectrum. The iodine lines provide calibration of both wavelength and the spectrometer point-spread function (Butler et al. 1996). While $20 \%$ of the starlight is absorbed by iodine, the cell's inclusion is worthwhile because the dense iodine absorption lines provide a permanent record of the wavelength scale and behavior of the spectrometer at the instant of each observation, producing long-term Doppler precision free of systematic errors to the level of $1 \mathrm{~m} \mathrm{~s}^{-1}$.

The velocity measurements are listed in Table 1 and shown in Figure 1 with different symbols for measurements made at Lick and Keck. We carried out a preliminary five-Keplerian fit to the combined velocities from both telescopes, allowing one extra parameter to be the difference in the velocity zero point from the two spectrometers, found to be $28.8 \pm 0.5 \mathrm{~m} \mathrm{~s}^{-1}$. Once established, we applied the $+28.8 \mathrm{~m} \mathrm{~s}^{-1}$ correction to the Keck data, putting the two spectrometers' measurements on the same velocity scale before showing them in Figure 1 and listing them in Table 1. The first 14 Doppler measurements made between 1989 and 1994 November typically have uncertainties of 8$10 \mathrm{~m} \mathrm{~s}^{-1}$, worse than most of the subsequent observations due to the unrepaired optics of the Hamilton spectrometer. Observations made at Lick since 1994 December have typical uncertainties of 3-5 m s${ }^{-1}$. At Keck, between 1999 and 2004, the typical Doppler uncertainty is $3 \mathrm{~m} \mathrm{~s}^{-1}$. In 2004 August the optics and CCD detector for HIRES were upgraded, reducing the Doppler errors. In 2004 November we began making three consecutive observations (and sometimes five) of $55 \mathrm{Cnc}$ to average 


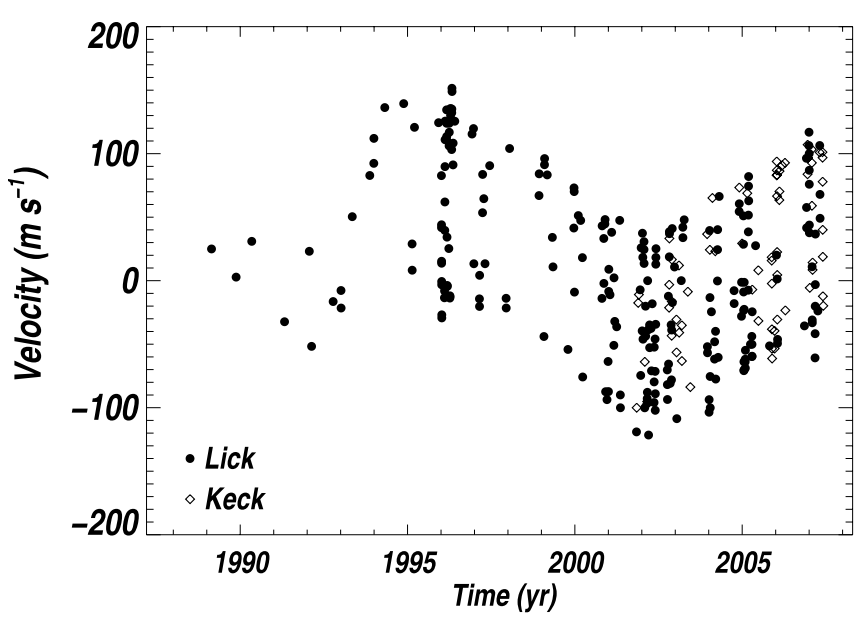

FIG. 1.-Measured velocities for $55 \mathrm{Cnc}$ from Lick and Keck obtained from 1989.1 to 2007.4. Data from Lick (filled circles) had errors of $\sim 10 \mathrm{~m} \mathrm{~s}^{-1}$ prior to 1994 and 3-5 m s${ }^{-1}$ thereafter. Data from Keck (open diamonds) had errors of $\sim 3 \mathrm{~m} \mathrm{~s}^{-1}$ prior to 2004 August and $1.0-1.5 \mathrm{~m} \mathrm{~s}^{-1}$ thereafter. The $14 \mathrm{yr}$ period from the outer planet and the short timescale variations from the 14.6 day planet are apparent to the eye.

over stellar $p$-mode oscillations that can add $1 \mathrm{~m} \mathrm{~s}^{-1}$ velocity noise to G8 V main-sequence stars (Kjeldsen et al. 2005). The resulting Doppler precision at Keck since 2004 August has been $1.0-1.5 \mathrm{~m} \mathrm{~s}^{-1}$.

\section{KEPLERIAN FITS TO DOPPLER MEASUREMENTS}

The Doppler measurements of $55 \mathrm{Cnc}$ were fit with a series of Keplerian models, each model having an increasing number of planets, beginning with the two well-established periods of 14.65 days and $14.7 \mathrm{yr}$ (Marcy et al. 2002). We polished all models with a Marquardt minimization of $\chi_{\nu}^{2}$ to establish the best-fit model. The weights assigned to each Doppler measurement are the inverse square of each measurement's uncertainty, which are approximated as the quadrature sum of the internal uncertainty in the Doppler measurement and the "jitter" that stems from photospheric motions and instrumental errors (Wright 2005). Experience with similar G8 main-sequence stars suggests that the combined astrophysical and instrumental jitter is $3 \mathrm{~m} \mathrm{~s}^{-1}$ at Lick and $1.5 \mathrm{~m} \mathrm{~s}^{-1}$ at Keck, both values being uncertain by $50 \%$. The jitter prediction is complicated by the high metallicity of $55 \mathrm{Cnc},[\mathrm{Fe} / \mathrm{H}]=+0.3$. The radiative transfer of $\mathrm{Ca}$ II $\mathrm{H}$ and $\mathrm{K}$ in $55 \mathrm{Cnc}$ will be different from that in solar-metallicity stars, because of higher line and continuous opacities, rendering the calibration of emission with stellar age, rotation, and jitter even more uncertain. However, the estimated rotation period of 39 days from periodicities in the $\mathrm{Ca}$ II $\mathrm{H}$ and $\mathrm{K}$ emission and the star's low rotational $v \sin i$ of $2.5 \mathrm{~km} \mathrm{~s}^{-1}$ confirm that the star has, at most, a modest level of magnetic activity, indicating correspondingly modest jitter with an upper limit of $4 \mathrm{~m} \mathrm{~s}^{-1}$. For this analysis, we adopt a jitter value of 1.5 and $3.0 \mathrm{~m} \mathrm{~s}^{-1}$ for Keck and Lick, respectively.

After fitting a model with the two well-established planets, we assessed the statistical significance of any periodicities remaining in the residuals to motivate addition of another planet to the model, as described in detail below. We determine false-alarm probabilities for peaks in the periodogram attributed to any additional planets by testing the null hypothesis that the current velocity residuals are merely incoherent noise. In such tests, the velocity residuals to our best-fit model are scrambled and their periodograms computed to assess the fraction of trials with scrambled residuals that have stronger peaks. This false-alarm

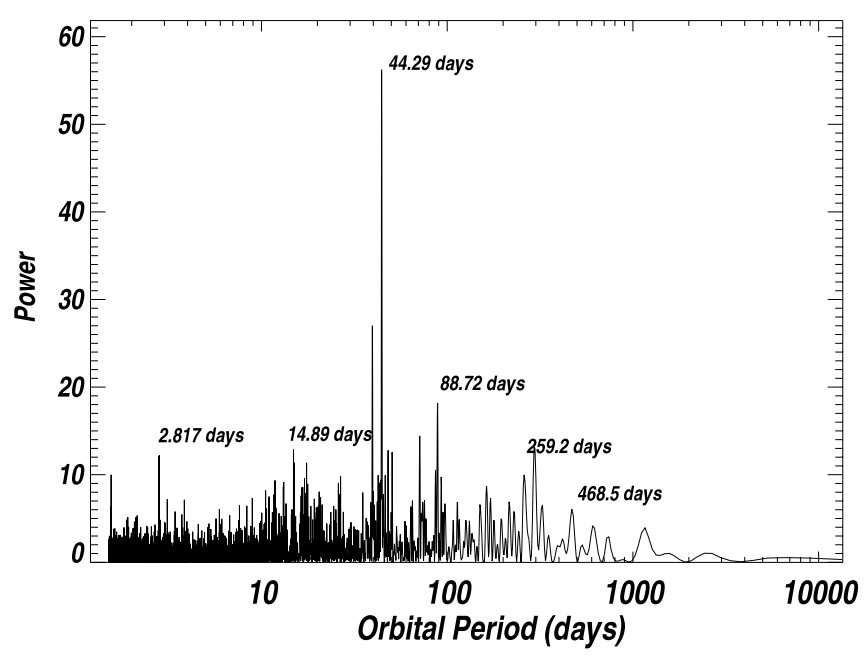

FIG. 2.- Periodogram of the residuals to a Keplerian model that contains only the two well-established planets with periods of 14.65 and 5200 days. The tall peak at $P=44.3$ days confirms the previously suspected planet with that period.

probability assessment makes few assumptions about the width or shape of the distribution of noise.

\subsection{The Three-Planet Model}

Our initial model consisted of the sum of two Keplerian orbits (no gravitational interactions) for the two planets having secure orbital periods of 14.65 days and $\sim 14.7 \mathrm{yr}$, both strongly supported by all of our past Doppler analyses of this star (Marcy et al. 2002). A two-planet fit yields periods of 14.65 days and $14.7 \mathrm{yr}$ and eccentricities of 0.002 and 0.06 for the two planets, respectively. The residuals have an $\mathrm{rms}$ of $11.28 \mathrm{~m} \mathrm{~s}^{-1}$ and $\left(\chi_{\nu}^{2}\right)^{1 / 2}$ of 3.42. An accurate model would have an $\mathrm{rms}$ on the order of the errors in the data plus jitter $\left(\sim 5 \mathrm{~m} \mathrm{~s}^{-1}\right)$ and $\left(\chi_{\nu}^{2}\right)^{1 / 2}$ near 1 . These large values of rms and $\left(\chi_{\nu}^{2}\right)^{1 / 2}$ indicate that the model is inadequate. The periodogram of the residuals (Fig. 2) exhibits a tall peak at a period of 44.3 days and a power of 55, clearly significant above the noise. This period corresponds to the orbit of the planet suspected in Marcy et al. (2002). This 44.3 day period is most likely caused by a third planet, as the only other explanation would be rotational variation from surface inhomogeneities. Such rotational explanations are ruled out both by the shorter stellar rotational period, 42.7 days, found in the photometry as shown in $\S 6$, and by the large velocity amplitude of $10.6 \mathrm{~m} \mathrm{~s}^{-1}$, which is never seen in such chromospherically quiet stars. Furthermore, photospheric features generally only survive for a few rotation periods of the star. It seems unlikely that surface inhomogeneities would persist for more than a decade and maintain rotational phase coherence.

A Levenberg-Marquardt minimization was used to find the best-fit orbital parameters for a three-planet Keplerian model with periods near 14.65 days, 44.34 days, and 14.7 yr. The best fit yielded residuals with an rms scatter of $8.62 \mathrm{~m} \mathrm{~s}^{-1}$ and $\left(\chi_{\nu}^{2}\right)^{1 / 2}=2.50$. This result represents an improved fit to the twoplanet model but is still clearly inadequate, not surprising as the model did not include a periodicity near 2.8 days as found by McArthur et al. (2004). Indeed, the periodogram of the residuals to the three-planet fit, shown in Figure 3, reveals two additional strong peaks near 2.8 and 260 days.

\subsection{The Four-Planet Model}

We proceeded to test a four-planet model by including a fourth planet with a period near 2.8 days (McArthur et al. 2004). The 


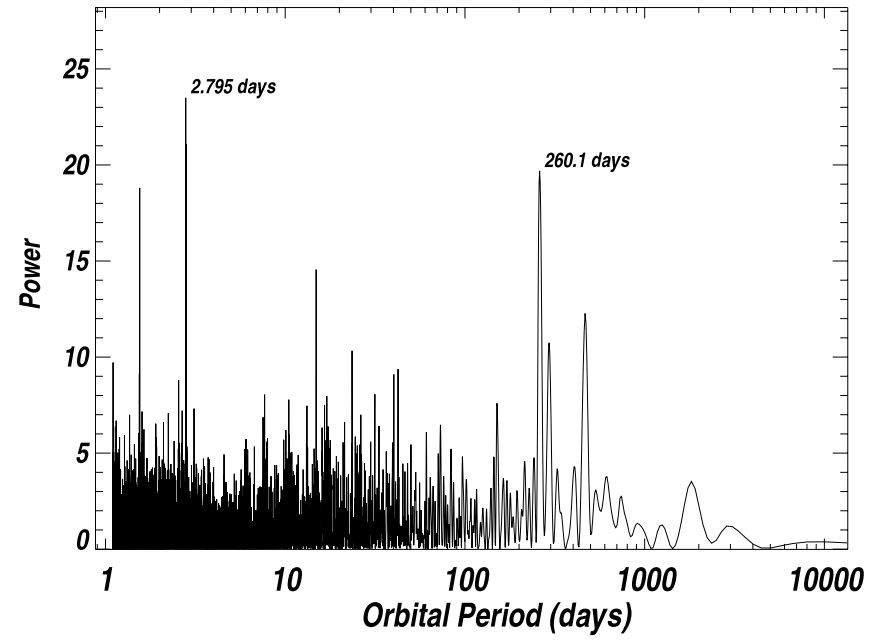

FIG. 3.- Periodogram of the residuals to a Keplerian model that contains three known planets with periods of $14.6,44.3$, and 5200 days. The tallest peaks are at 2.81 and 260 days, suggesting the existence of real periodicities in the velocities. The peak at $\sim 1.5$ days is an alias of the 2.8 day peak, and the peak at 460 days is an alias of that at 260 days that disappears after modeling all five planets.

best-fit four-planet model gave periods of 2.81 days, 14.65184 days, 44.32 days, and $14.4 \mathrm{yr}$, all with eccentricities less than 0.3 . The residuals have rms of $7.87 \mathrm{~m} \mathrm{~s}^{-1}$ and $\left(\chi_{\nu}^{2}\right)^{1 / 2}=2.12$, both representing a significant improvement over the three-planet model. (In computing both the rms and $\chi_{\nu}^{2}$, the denominator was appropriately diminished by the greater number of free parameters, i.e., five per planet.) Thus, both the periodogram in Figure 3 and the superior fit with four planets offer support for the existence of the planet with 2.81 days, corresponding to the planet with $P=2.808 \pm 0.002$ days in McArthur et al. (2004).

However, this four-planet model remains inadequate for two reasons. The residuals reveal a poor fit with $\left(\chi_{\nu}^{2}\right)^{1 / 2}=2.12$ and an rms of $7.87 \mathrm{~m} \mathrm{~s}^{-1}$, larger than explainable by the Doppler errors and jitter. In addition, a periodogram of the residuals reveals a peak at a period of 260.1 days, as shown in Figure 4, and some additional smaller peaks.

\subsection{Assessing the Periodicity near 260 days}

The periodogram peak near 260 days (Fig. 4) in the residuals to the four-planet model could be spurious, caused by fluctuations arising from photon-limited Doppler errors in the spectra or by aliases in the window function of the sampling times. The CCD detector at Lick Observatory has been upgraded four times in the past 18 years, which could produce discontinuities of 1$2 \mathrm{~m} \mathrm{~s}^{-1}$ in their zero points and even create an alias. Such abrupt, onetime instrumental changes should not produce periodicities. Nonetheless, to check for such effects, the four-planet Keplerian model was fit separately to the Lick and Keck velocities.

The 250 velocities from Lick against only 70 from Keck cause the periodogram in Figure 4 to be heavily weighted toward the Lick measurements. The prominent period at 260 days certainly reflects the Lick velocities more than those from Keck, leaving open the question of independent confirmation of the 260 day period in the Keck data. We fit a four-planet model to the $70 \mathrm{Keck}$ velocities alone. The Keck velocities offer higher precision $\left(\sim 1.5 \mathrm{~m} \mathrm{~s}^{-1}\right)$ than those from Lick but carry the disadvantage of a duration of only $5.5 \mathrm{yr}$.

The four-planet fit to the Keck velocities alone yielded residuals with rms $=4.3 \mathrm{~m} \mathrm{~s}^{-1}$ and $\left(\chi_{\nu}^{2}\right)^{1 / 2}=2.59$. The periodogram of the residuals is shown in Figure 5, and it reveals a peak at a period of 266 days with a power of 7.7. There is no significant

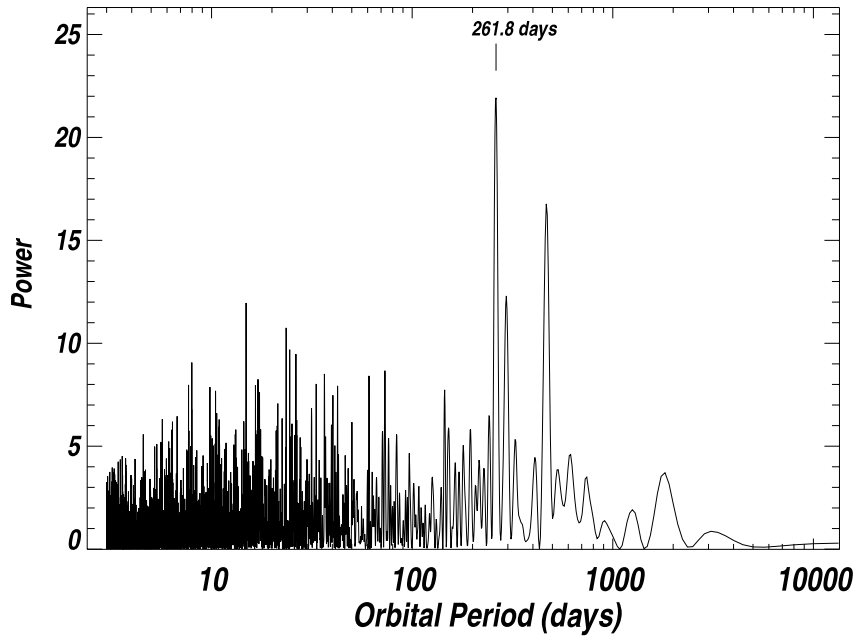

FIG. 4.- Periodogram of the residuals to a Keplerian model that contains the four previously suspected planets with periods near $2.817,14.65,44.3$, and 5200 days. The periodogram exhibits a peak at 260.0 days, caused by the prospective fifth planet in the system. The smaller peak to its right at 460 days is an alias.

power at any other periods. The power in the 266 day peak is higher than all peaks for periods between 1 and 3000 days. Importantly, the peak at 266 days is roughly twice as high as the noise peaks. Although this peak is not overwhelming by itself, the independent occurrence of a periodicity near 265 days in the Keck velocities along with the similar period found in the Lick velocities supports the reality of that period and argues against systematic errors as the cause.

One might be concerned that the Keck velocities yielded such a modest peak at $\sim 266$ days (Fig. 5) as compared to the relatively strong peak in the Lick data (Fig. 4). We addressed this concern by augmenting the Keck velocities with artificial velocities corresponding to a planet having a period of 260 days in a Keplerian orbit that causes a semiamplitude of $K=4.4 \mathrm{~m} \mathrm{~s}^{-1}$. The idea is that if the power in the periodogram doubles, then the modest peak in Figure 5 is probably reasonable. We performed a fourplanet fit and computed the periodogram of the residuals. A peak at $P=261$ days was seen with a power of 13 , roughly double the

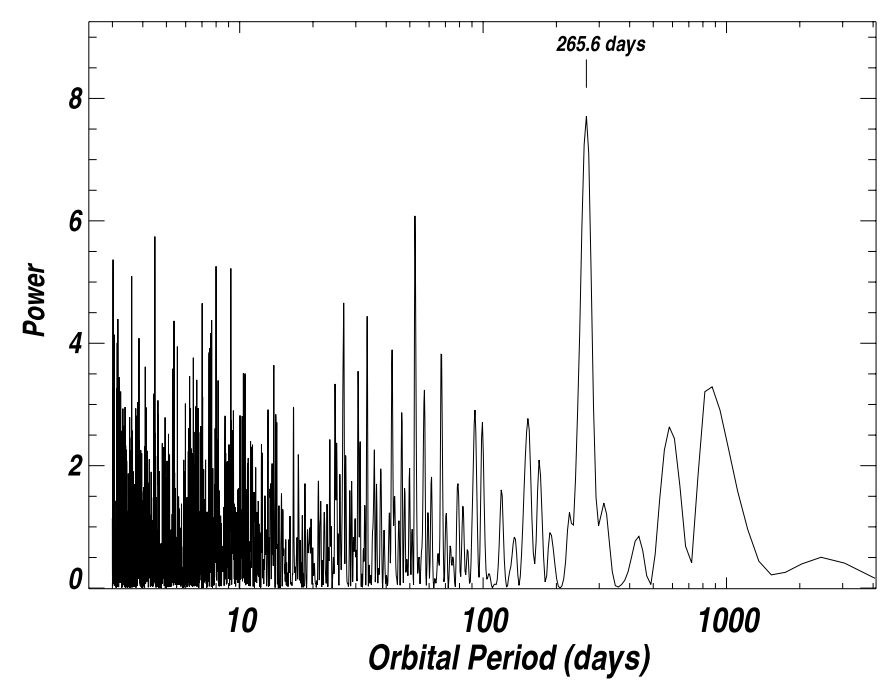

FIG. 5.- Periodogram of the residuals to a four-planet Keplerian model, as in Fig. 4, but fit to the Keck velocities only. The tallest peak is at a period of 265.6 days, nearly the same as that emerging from the Lick data. The modest peak power of only 7.7 is consistent with the limited time sampling and duration of the Keck observations. No other period is compelling between periods of 1 and 3000 days. 


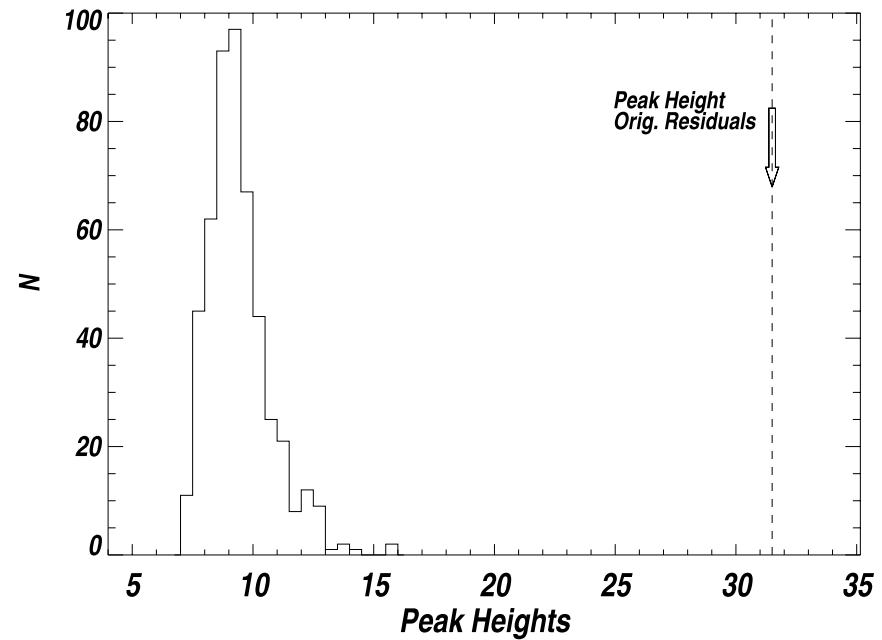

FIG. 6.-Test of the false-alarm probability of the 260 day periodicity seen in the combined velocities from both Lick and Keck (Fig. 4). The residuals to a fourplanet model were scrambled 500 times, yielding a histogram of the highest power in the periodograms. The power of 31.8 from the original residuals (Fig. 4) is greater than that from all 500 trials, implying a false-alarm probability for the 260 day period of less than 0.002 , suggesting that it is real.

power of the peak that emerged from the original velocities. Thus, the 266 day peak in the periodogram from the original Keck velocities (Fig. 5) constitutes a confirmation of the $\sim 265$ day planet seen in Figure 4 having that period and amplitude. Of course, the Lick velocities alone also exhibit the 260 day peak independently.

We also checked to see if the 260 day signal might be an alias of the possible 470 day peak seen in Figure 4 . We fit the combined Lick and Keck velocities with a five-planet model having a fifth planet with a period near 470 days instead of near 260 days. The periodogram of the residuals to this five-planet model still has a strong peak with period near 263 days, with a power of 19 . Apparently the period at 260 days does not vanish by including a 470 day period in the model and thus is not an alias of it.

We assessed the probability that the 260 day signal was caused by chance fluctuations in the velocities by performing a conservative false-alarm probability test. We fit the combined velocities with only a four-planet model and tested the null hypothesis that no periodicity near 260 days actually exists in the residuals, implying that the peak is due merely to noise. We scrambled the residuals to the four-planet fit but kept the times of observation the same, and recomputed the periodogram for each of 1000 trials. We recorded the power of the tallest peak in the periodogram from each of 1000 trials. The histogram of those peak powers is shown in Figure 6. The typical peaks from the scrambled residuals have powers of $7-13$, with the tallest being 15 . In contrast, the periodogram of the original residuals had a peak height of 31.8, shown both in Figure 4 and as the vertical dashed line in Figure 6. Thus, the null hypothesis (that the residuals have no coherence) is unlikely and the associated false-alarm probability of the peak at 260 days is less than 0.001 , indicating that the periodicity is real.

The analysis above strongly supports the existence of a planet with a period of 260 days. The period of 260 days does not correspond to any known timescale of stellar interiors or atmospheres, nor to the rotation period of the star, which is 42.7 days (see below). Thus, a plausible interpretation is a planet with a period near $P=260$ days, making it the fifth planet in the $55 \mathrm{Cnc}$ system.

\subsection{The Five-Planet Model with a 260 day Planet}

We constructed a Keplerian model that included a fifth planet having a period near 260 days. A best-fit model to the combined Lick and Keck velocities was found easily, yielding five periods of 14.65162, 44.344, 5218, 2.817, and 260.0 days (see Table 2). The residuals have $\mathrm{rms}=6.74 \mathrm{~m} \mathrm{~s}^{-1}$ and $\left(\chi_{\nu}^{2}\right)^{1 / 2}=1.67$ (including the jitter in the expected variance). The values of the rms and $\left(\chi_{\nu}^{2}\right)^{1 / 2}$ are $15 \%$ and $20 \%$ lower, respectively, than the corresponding diagnostics of the four-planet model. Table 3 gives the rms and $\left(\chi_{\nu}^{2}\right)^{1 / 2}$ for all multiplanet models considered in this paper, showing the significant improvement with each additional planet. This major improvement in the quality of the fit of 320 measurements, coming from a fifth planet with its five additional free parameters, indicates that the new model has considerable merit. The five-planet model containing the 260 day planet is clearly superior to the four-planet model. The period agrees with that found by Wisdom (2005) from a periodogram analysis of our earlier, published velocities from Lick Observatory.

As this model contains a proposed planet with $P=260$ days, we present in Table 2 all of the orbital parameters for all five planets self-consistently computed with a Levenberg-Marquardt least-squares algorithm. Considerable trial and error with various starting guesses for the 26 free parameters was carried out to ensure that the least-squares search began near the deepest minimum. The $\chi_{\nu}^{2}$ fit was virtually unchanged for orbital eccentricities between 0.0 and 0.4 for the 260 day planet. This is not surprising since the amplitude of the planet is comparable to the single measurement precision for most of our data. Although the orbit is consistent with circular, we adopted an intermediate eccentricity of $0.2 \pm 0.2$ to indicate the indistinguishable range of eccentricity. The best-fit parameters for the 260 day planet are $e=0.2 \pm 0.2$ and $K=4.879 \pm 0.6 \mathrm{~m} \mathrm{~s}^{-1}$, implying $M \sin i=0.144 \pm 0.04 M_{\text {Jup }}$.

The innermost planet has $P=2.81705 \pm 0.0001$ days, $e=$ $0.07 \pm 0.06, K=5.07 \pm 0.53 \mathrm{~m} \mathrm{~s}^{-1}$, and $M \sin i=0.034 M_{\mathrm{Jup}}=$ $10.8 M_{\oplus}$. In comparison, McArthur et al. (2004) found the inner planet to have $P=2.808 \pm 0.002$ days, $e=0.174 \pm 0.127$, $K=6.67 \mathrm{~m} \mathrm{~s}^{-1}$, and $M \sin i=0.045 M_{\text {Jup }}=14.2 M_{\oplus}$.

There is no question that the planet with $P=2.817$ days is the planet previously identified by McArthur et al. (2004). We note

TABLE 2

Orbital Parameters for the Five-Planet Model

\begin{tabular}{|c|c|c|c|c|c|c|c|}
\hline Planet $^{\mathrm{a}}$ & $\begin{array}{l}\text { Period } \\
\text { (days) }\end{array}$ & $T_{p}$ & $e$ & $\begin{array}{c}\omega \\
(\operatorname{deg})\end{array}$ & $\begin{array}{c}K \\
\left(\mathrm{~m} \mathrm{~s}^{-1}\right)\end{array}$ & $\begin{array}{c}M \sin i \\
\left(M_{\text {Jup }}\right)\end{array}$ & $\begin{array}{c}a \\
(\mathrm{AU})\end{array}$ \\
\hline e.. & $2.81705 \pm 0.0001$ & $249999.83643 \pm 0.0001$ & $0.07 \pm 0.06$ & $248.9 \pm 38$ & $5.07 \pm 0.53$ & $0.034 \pm 0.0036$ & $0.038 \pm 1.0 \times 10^{-6}$ \\
\hline 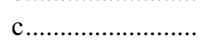 & $44.3446 \pm 0.007$ & $2449989.3385 \pm 3.3$ & $0.086 \pm 0.052$ & $77.9 \pm 29$ & $10.18 \pm 0.43$ & $0.169 \pm 0.008$ & $0.240 \pm 4.5 \times 10^{-5}$ \\
\hline $\mathrm{f}$ & $260.00 \pm 1.1$ & $2450080.9108 \pm 1.1$ & $0.2 \pm 0.2(\mathrm{f})$ & $181.1 \pm 60$ & $4.879 \pm 0.6$ & $0.144 \pm 0.04$ & $0.781 \pm 0.007$ \\
\hline $\mathrm{d}$ & $5218 \pm 230$ & $2452500.6 \pm 230$ & $0.025 \pm 0.03$ & $181.3 \pm 32$ & $46.85 \pm 1.8$ & $3.835 \pm 0.08$ & $5.77 \pm 0.11$ \\
\hline
\end{tabular}

${ }^{a}$ Planets are listed in order of increasing orbital period; however, the planet designations, $b-f$, correspond to the chronological order of their discovery. 
TABLE 3

Summary of Improvements In RMS and $\chi_{\nu}^{2}$ Fits

\begin{tabular}{|c|c|c|}
\hline Planet & $\begin{array}{c}\mathrm{rms} \\
\left(\mathrm{m} \mathrm{s}^{-1}\right)\end{array}$ & $\left(\chi_{\nu}^{2}\right)^{1 / 2}$ \\
\hline $\mathrm{b}, \mathrm{c} \ldots \ldots \ldots \ldots \ldots \ldots \ldots$ & 11.28 & 3.42 \\
\hline $\mathrm{b}, \mathrm{c}, \mathrm{d}$ & 8.62 & 2.50 \\
\hline 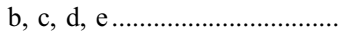 & 7.87 & 2.12 \\
\hline 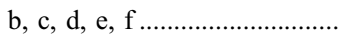 & 6.74 & 1.67 \\
\hline
\end{tabular}

that the new minimum mass $M \sin i=10.8 M_{\oplus}$ is lower than the 14.2 $M_{\oplus}$ previously reported in McArthur et al. These differences are not surprising as some of the excess velocity variation previously left to be absorbed by the four known planets is now accounted for by the fifth planet.

The phase-folded velocities for the 260 day planet are shown in Figure 7 after subtracting the sum of the computed velocities of the other four planets from the measured velocities. The orbital eccentricity has been fixed to 0.2 . The resulting residual velocities are plotted versus orbital phase and shown in Figure 7. The residuals reveal the 260 day period that had been detected in the periodogram and the Keplerian model is overplotted. The scatter has an rms of $6.74 \mathrm{~m} \mathrm{~s}^{-1}$. The error bars shown in Figure 7 include the quadrature sum of the internal errors (typically $2 \mathrm{~m} \mathrm{~s}^{-1}$ for Keck and $4 \mathrm{~m} \mathrm{~s}^{-1}$ for Lick) and the jitter $\left(1.5 \mathrm{~m} \mathrm{~s}^{-1}\right.$ for Keck and $3 \mathrm{~m} \mathrm{~s}^{-1}$ for Lick). Thus, the scatter of $6.74 \mathrm{~m} \mathrm{~s}^{-1}$ is only slightly larger than the known internal errors and expected jitter (astrophysical and instrumental). Figure 8 shows the phased plot of the 260 day planet with a best-fit orbital eccentricity of 0.16 .

\section{RESIDUAL PLANETS}

Several explanations for the modest $6.74 \mathrm{~m} \mathrm{~s}^{-1}$ scatter in the residuals are possible. Perhaps we are underestimating our internal errors. Perhaps the jitter for this metal-rich star is somewhat higher than the average for G8 main-sequence stars. Or perhaps there are other planets that cause a sufficiently low signal that they are not apparent in the periodograms but nonetheless add a few meters per second of "noise" to the velocities.

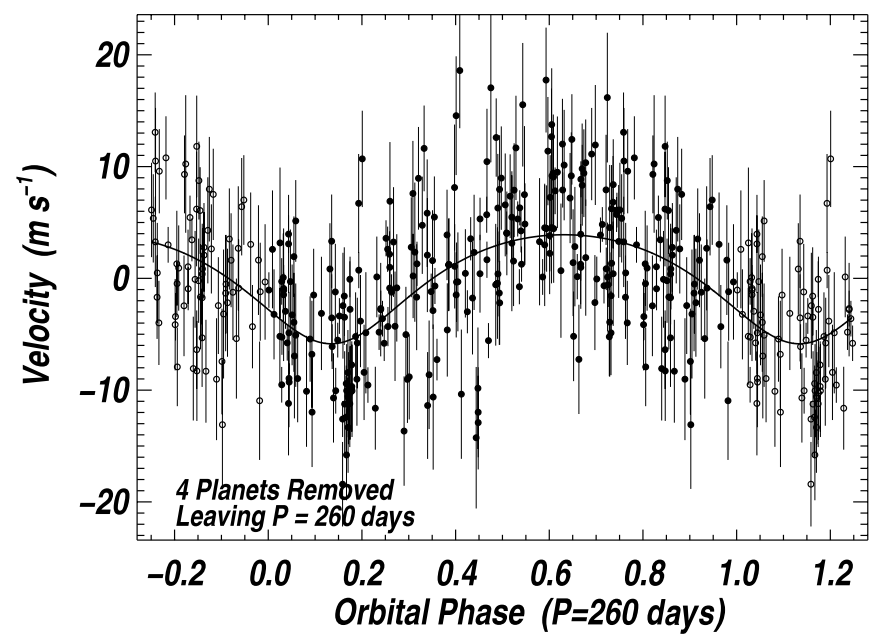

FIG. 7.-Residual velocities vs. orbital phase (for $P=260$ days) after the velocities induced by the four other planets are subtracted. The orbital parameters were established with a simultaneous five-planet Keplerian fit to all Doppler measurements. The residual velocities reveal the periodic variation associated with the new planet. The solid line shows the Keplerian curve of the 260 day planet alone, with eccentricity frozen to 0.2 . The planet's minimum mass is $45 M_{\oplus}$, and the semimajor axis is $0.78 \mathrm{AU}$.

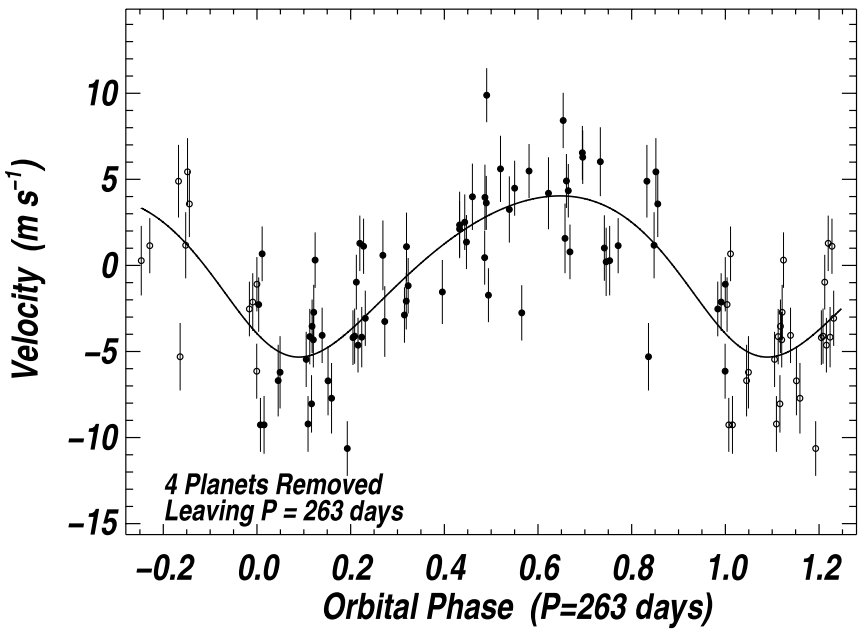

FIG. 8. - Velocity of the new planet vs. orbital phase, as in Fig. 7, but for Keck velocities only. The periodicity near 260 days is apparent independently in the Keck velocities. The best fit to the Keck velocities yields an eccentricity of $e=0.16$, only marginally significant.

We assessed the detectability of a hypothetical sixth planet by adding the velocities that would be induced by it to the observed velocities. We fit a five-planet model to these augmented velocities, allowing all 26 parameters to float. We searched the periodogram of the residuals for peaks that loom above those arising in the five-planet fit of the actual velocities (Fig. 9). Such peaks would have been identified as a candidate sixth planet. We considered orbital periods from 300 to 4000 days and determined the minimum $M \sin i$ that produced a peak $50 \%$ above any of the peaks in the actual periodogram (i.e., above a power of 15).

The minimum detectable mass of a hypothetical sixth planet is a sensitive function of its period and phase as those parameters determine how easily the signal can be absorbed in the five-planet model, avoiding detection. Neighboring periods differing by a mere few percent can produce periodogram peaks differing by a factor of 2 simply due to differing commensurability with the five existing planets. A sixth planet in a mean motion resonance is particularly capable of avoiding detection in the face of the five existing planets. Such fine structure aside, the simulations

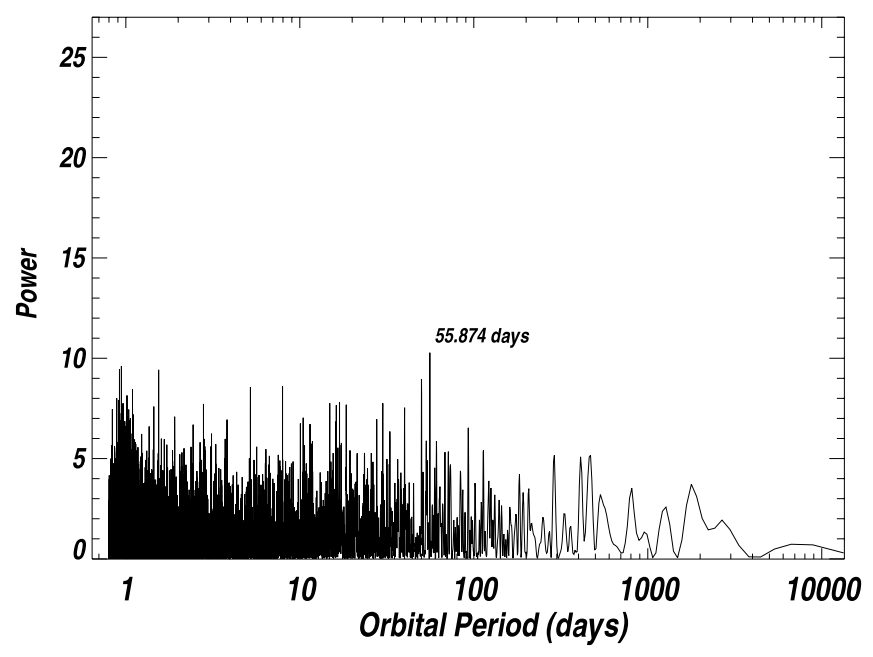

FIG. 9.- Periodogram of the velocity residuals to a Keplerian model that contains five planets including the prospective new planet at $P \approx 260$ days. Velocities from both Lick and Keck are included. The peak that had been apparent at 260 days in the residuals to a four-planet model (Figs. 4 and 5) has vanished due to its inclusion in the five-planet model. No other compelling periods are apparent. 
TABLE 4

Orbital Parameters from Self-Consistent Dynamical Fit

\begin{tabular}{|c|c|c|c|c|c|c|c|}
\hline $\begin{array}{c}\text { Planet } \\
\text { (1) }\end{array}$ & $\begin{array}{c}\text { Period } \\
\text { (days) } \\
(2)\end{array}$ & $\begin{array}{c}T_{p} \\
(\mathrm{JD}-2,440,000) \\
(3)\end{array}$ & $\begin{array}{c}e \\
(4)\end{array}$ & $\begin{array}{c}\omega \\
(\mathrm{deg}) \\
(5)\end{array}$ & $\begin{array}{c}K \\
\left(\mathrm{~m} \mathrm{~s}^{-1}\right) \\
(6)\end{array}$ & $\begin{array}{c}M \sin i \\
\left(M_{\mathrm{Jup}}\right) \\
(7)\end{array}$ & $\begin{array}{c}a \\
(\mathrm{AU}) \\
(8)\end{array}$ \\
\hline $55 \mathrm{Cnc} \mathrm{b}^{\mathrm{a}}$. & 14.651262 & 7572.0307 & 0.0159 & 164.001 & 71.84 & 0.8358 & 0.115 \\
\hline 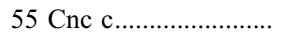 & 44.378710 & 7547.5250 & 0.0530 & 57.405 & 10.06 & 0.1691 & 0.241 \\
\hline $55 \mathrm{Cnc} d \mathrm{f} \ldots \ldots \ldots \ldots \ldots \ldots \ldots \ldots \ldots \ldots$ & 5371.8207 & 6862.3081 & 0.0633 & 162.658 & 47.20 & 3.9231 & 5.901 \\
\hline $55 \mathrm{Cnc}$ f.... & 260.6694 & 7488.0149 & 0.0002 & 205.566 & 4.75 & 0.1444 & 0.785 \\
\hline
\end{tabular}

${ }^{\mathrm{a}}$ Epoch $=\mathrm{JD} 2,447,578.730,\left(\chi^{2}\right)^{1 / 2}=2.012$ (without jitter included), and rms $=7.71 \mathrm{~m} \mathrm{~s}^{-1}$.

can be characterized as follows. For orbital periods of 300 850 days, a sixth planet with $M \sin i$ below $50 M_{\oplus}$ would have eluded detection as the periodogram peaks would not have loomed even $50 \%$ above the noise. For periods $850-1500$ days, a sixth planet could avoid detection by having $M \sin i$ below $100 M_{\oplus}$. For periods $1750-4000$ days, planets below $250 M_{\oplus}$ would elude detection. Thus, such planets could exist around $55 \mathrm{Cnc}$ and yet have avoided detection by our current 18 years of Doppler measurements. Indeed, several such planets could exist in the large gap between periods of 260 days and $13 \mathrm{yr}$ and probably maintain dynamical stability.

\section{DYNAMICAL SIMULATIONS OF THE MULTIPLANET SYSTEM}

The models in this paper are based on the approximation that the planetary orbits are Keplerian ellipses. In actuality, the radial velocity variation of the parent star over nearly two decades of observation is also affected by the mutual gravitational perturbations between the planets. As a concrete example, one can interpret the five-planet fit in Table 2 as describing a set of oscillating orbital elements at the epoch JD 2,447,578.730 of the first radial velocity observation. By making a choice of epoch, one creates a unique initial condition for a six-body integration of Newton's equations of motion. When this integration is carried out, one finds radial velocity deviations of $\Delta V>25 \mathrm{~m} \mathrm{~s}^{-1}$ in comparing Keplerian and Newtonian models at epochs near JD 2,454,000.

These deviations arise primarily from the orbital precessions of planets b, c, and d that occur in the Newtonian model that are absent from the Keplerian model. Because the orbits are nearly circular, a Keplerian five-planet fit can, however, compensate for nearly all of the precession through small adjustments to the orbital periods.

It is likely that one can obtain an improved $\chi^{2}$ by adopting a self-consistent $N$-body model for the stellar reflex velocity (e.g., Rivera et al. 2005). In addition to lowering the rms of the fit, a definitive model of this type allows for the correct characterization of the possible $3: 1$ resonant relationship between planets $\mathrm{b}$ and $\mathrm{c}$, and can therefore give important clues on the formation history of the system. Adopting the Keplerian fit in Table 2 as an initial guess, we used Levenberg-Marquardt minimization to obtain a self-consistent five-planet dynamical fit to the radial velocity data sets. The resulting orbital parameters of our dynamical fit are all quite similar to their corresponding values in the Keplerian model and are listed in Table 4. Our dynamical fit has $\left(\chi^{2}\right)^{1 / 2}=2.012$ (without including any jitter) and $\mathrm{rms}=$ $7.712 \mathrm{~m} \mathrm{~s}^{-1}$. A more computationally expensive search should be able to find orbital parameters that provide a slight improvement to these values. We leave such an analysis to future work.
In order to assess the dynamical stability of our five-planet model, we adopt the self-consistent orbital elements in Table 4 and integrate the system forward for 1 Myr from epoch JD 2,447,578.730. We used a Bulirsch-Stoer integrator (Press et al. 1992). The system remained stable throughout a 1 Myr integration. The evolution of the five planetary eccentricities during a representative $2.5 \times 10^{4} \mathrm{yr}$ interval is shown in Figure 10 . As is true throughout the $1 \mathrm{Myr}$ integration, the eccentricity variations experienced by all five planets are quite modest, and the system appears likely to be dynamically stable for long periods.

It is interesting to note that during the course of the numerical integration, the $3: 1$ resonant arguments for planets $b$ and $c$ are all circulating. This indicates that planets $\mathrm{b}$ and $\mathrm{c}$ do not currently participate in a low-order mean motion resonance, despite the near commensurability of their orbital periods.

We have computed the eccentricity variations that result when the system is modeled using a secular perturbation theory that includes terms up to second order in eccentricity (see, e.g., Murray \& Dermott 1999) and which includes the leading-order effects of general relativity as outlined by Adams \& Laughlin (2006). The results are quite similar to those in Figure 10. This indicates that the bulk of the planet-planet interactions in the system can be accounted for with a simple second-order theory, thus improving the likelihood that the configuration of planets can remain dynamically stable for the lifetime of the star.

\section{PHOTOMETRY OF 55 CANCRI}

We have used the T8 $0.8 \mathrm{~m}$ automatic photometric telescope (APT) at Fairborn Observatory to obtain high-precision photometry

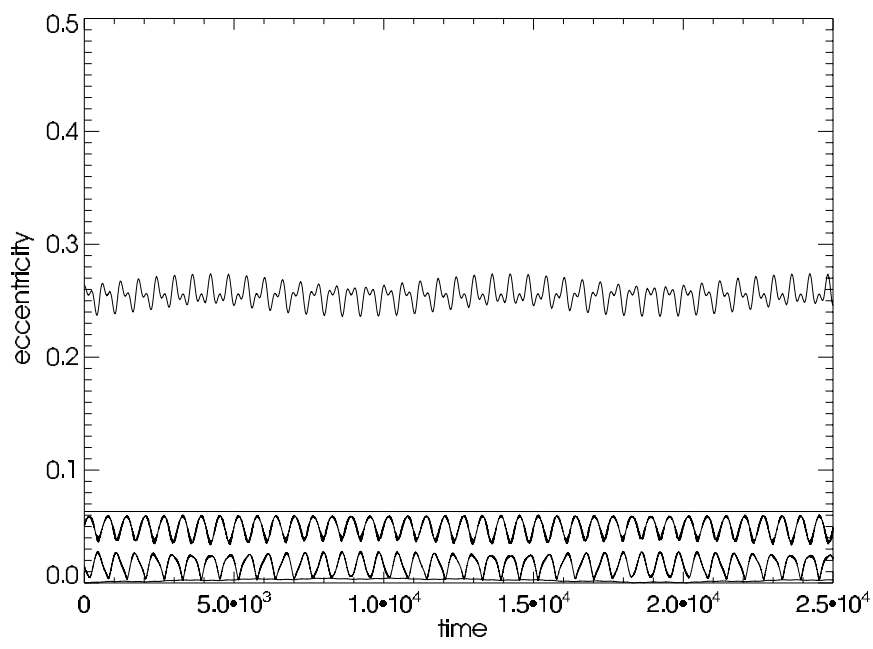

FIG. 10.-Eccentricity variations arising from an $\mathrm{N}$-body numerical integration of the five-planet model listed in Table 2. 
of 55 Cnc during 11 observing seasons between 1996 November and 2007 April. The T8 APT is one of several automated telescopes at Fairborn dedicated to observing long-term, low-amplitude brightness variations in solar-type stars associated with stellar magnetic cycles as well as to measuring short-term, low-amplitude variations caused by rotational modulation in the visibility of surface magnetic features (Henry 1999). APT photometry of planetary candidate stars helps to establish whether observed radial velocity variations are caused by stellar activity or planetaryreflex motion, and direct measurements of stellar rotation periods provide good age estimates of the planetary systems (e.g., Henry et al. 2000). Queloz et al. (2001) and Paulson et al. (2004) have published several examples of periodic radial velocity variations in solar-type stars caused by photospheric spots and plages. The APT observations are also useful to search for transits of the planetary companions. The rare transiting systems allow direct determination of basic planetary parameters such as mass, radius, and mean density and so provide observational constraints on models of planetary composition and internal structure (e.g., Sato et al. 2005). Bright transiting systems enable detailed follow-up studies of exoplanet atmospheres (e.g., Richardson et al. 2007). Finally, monitoring a planetary host star's long-term luminosity variations provides a measure of the star's climate forcing ability on its system of planets (e.g., Hall et al. 2007).

The T8 APT is equipped with a two-channel precision photometer employing two EMI 9124QB bi-alkali photomultiplier tubes to make simultaneous measurements in the Strömgren $b$ and $y$ passbands. The APT measures the difference in brightness between a program star and one or more nearby constant comparison stars; the primary comparison star used for $55 \mathrm{Cnc}$ is HD $76572(V=6.28, B-V=0.42$, F6 IV-V). The Strömgren $b$ and $y$ differential magnitudes are corrected for differential extinction with nightly extinction coefficients and transformed to the Strömgren system with yearly mean transformation coefficients. Finally, the $\Delta b$ and $\Delta y$ observations are combined into a single $\Delta(b+y) / 2$ passband to increase the photometric precision. The external precision of a single differential magnitude is typically around $0.0015 \mathrm{mag}$ for the T8 APT, as determined from pairs of constant stars. Further details on the telescope, photometer, observing procedures, and data reduction techniques can be found in Henry (1999).

The complete 11-year set of differential magnitudes computed with the primary comparison star is plotted in the top panel of Figure 11. Intercomparison of the primary comparison star with two secondary comparisons (HD 77190: $V=6.07, B-V=$ 0.24 , A8 Vn; HD 79929: $V=6.77, B-V=0.41, \mathrm{~F} 6 \mathrm{~V}$ ) revealed that the annual means of the primary comparison vary over a range of $0.003 \mathrm{mag}$ from year to year. Rather than switch to one of the more stable secondary comparison stars, we have instead normalized the 11 seasons with the primary comparison so they all have the same annual mean. This was done because the secondary comparison stars have been used only for the past seven years. The normalization removes any long-term variation in the primary comparison star as well as in $55 \mathrm{Cnc}$, but this improves the sensitivity of our transit search described below for orbital periods under $1 \mathrm{yr}$. After normalization, outliers exceeding $3 \sigma$ were removed. The final data set in the top panel of Figure 11 contains 1349 nightly observations; the standard deviation of an individual observation from the normalized mean is $0.0017 \mathrm{mag}$.

The 0.0017 mag standard deviation of the full data set is only slightly greater than the nominal measurement precision of 0.0015 mag but suggests that low-amplitude, short-term intrinsic variability might be present at times in $55 \mathrm{Cnc}$. (Long-term var-
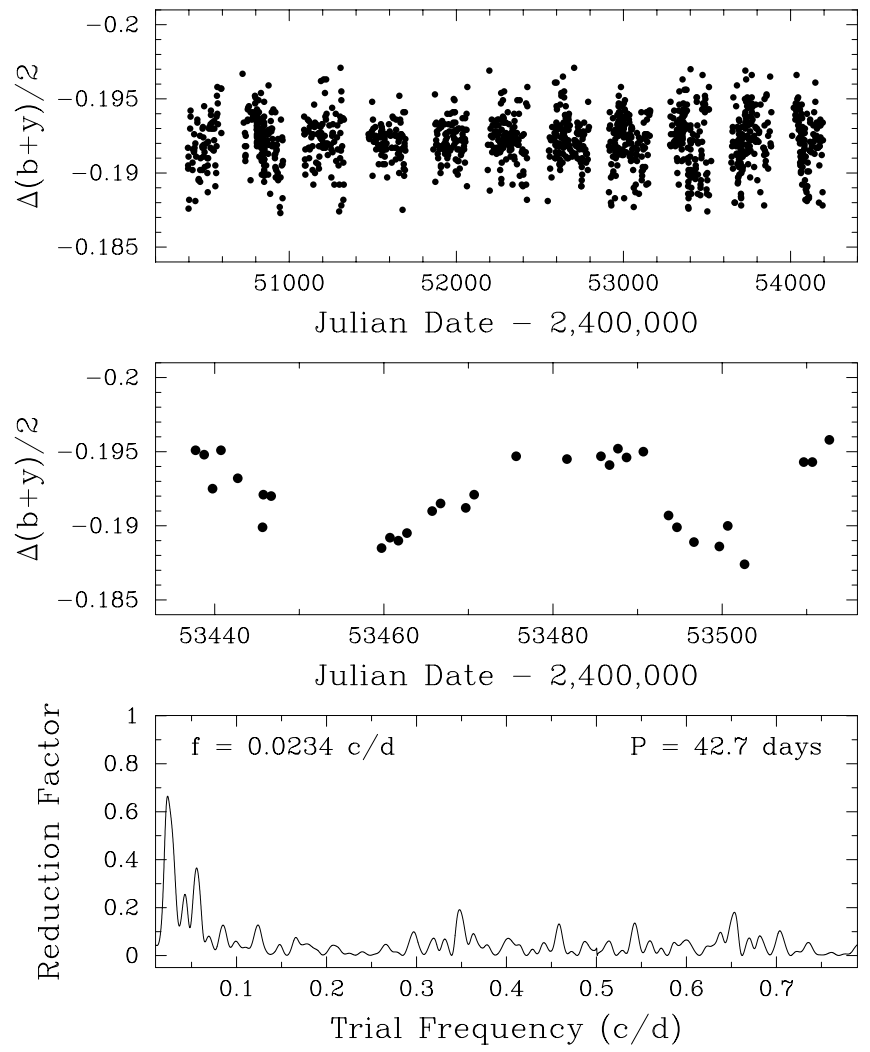

FIG. 11.-Eleven-year Strömgren photometric data set of $55 \mathrm{Cnc}$ (top). The data have been normalized so that the annual means are identical. A portion of the ninth observing season (middle) shows coherent photometric variability in $55 \mathrm{Cnc}$ due to rotational modulation in the visibility of starspots. The power spectrum of the data in the middle panel (bottom) reveals the star's rotation period of $42.7 \pm 2.5$ days.

iability has been removed by the normalization.) We searched each annual set of measurements for evidence of coherent, lowamplitude variability that might be the result of rotational modulation in the visibility of starspots. The middle panel of Figure 11, which shows photometry from a portion of the ninth observing season, exhibits the clearest example of coherent variability in the data set. Two cycles of brightness variation are visible with an amplitude of approximately $0.006 \mathrm{mag}$. We interpret this as evidence for a small starspot region (covering less than $1 \%$ of the star's visible surface) that has survived for two rotation cycles of the star. A power spectrum of the observations in the middle panel is computed with the method of Vaníček (1971) and shown in the bottom panel of Figure 11. This gives a period of $42.7 \pm$ 2.5 days, which we interpret to be the stellar rotation period. This confirms the rotation period of $55 \mathrm{Cnc}$ reported by Henry et al. (2000), who used rotational modulation of the $\mathrm{Ca}$ II $\mathrm{H}$ and $\mathrm{K}$ flux measured by the HK Project at Mount Wilson Observatory (Baliunas et al. 1998).

We searched the photometric data for evidence of transits of the four inner planets; the results are summarized in Table 4 and plotted in Figure 12. We first computed the semiamplitudes of the light curves (col. [3]) with least-squares sine fits of the complete data set phased to the four shortest radial velocity periods. The resulting amplitudes are all extremely small and consistent with zero. These very tight limits on photometric variability on the radial velocity periods clearly support planetary-reflex motion as the cause of the radial velocity variations. While our measured 42.7 day rotation period is consistent with the 44.35 day 


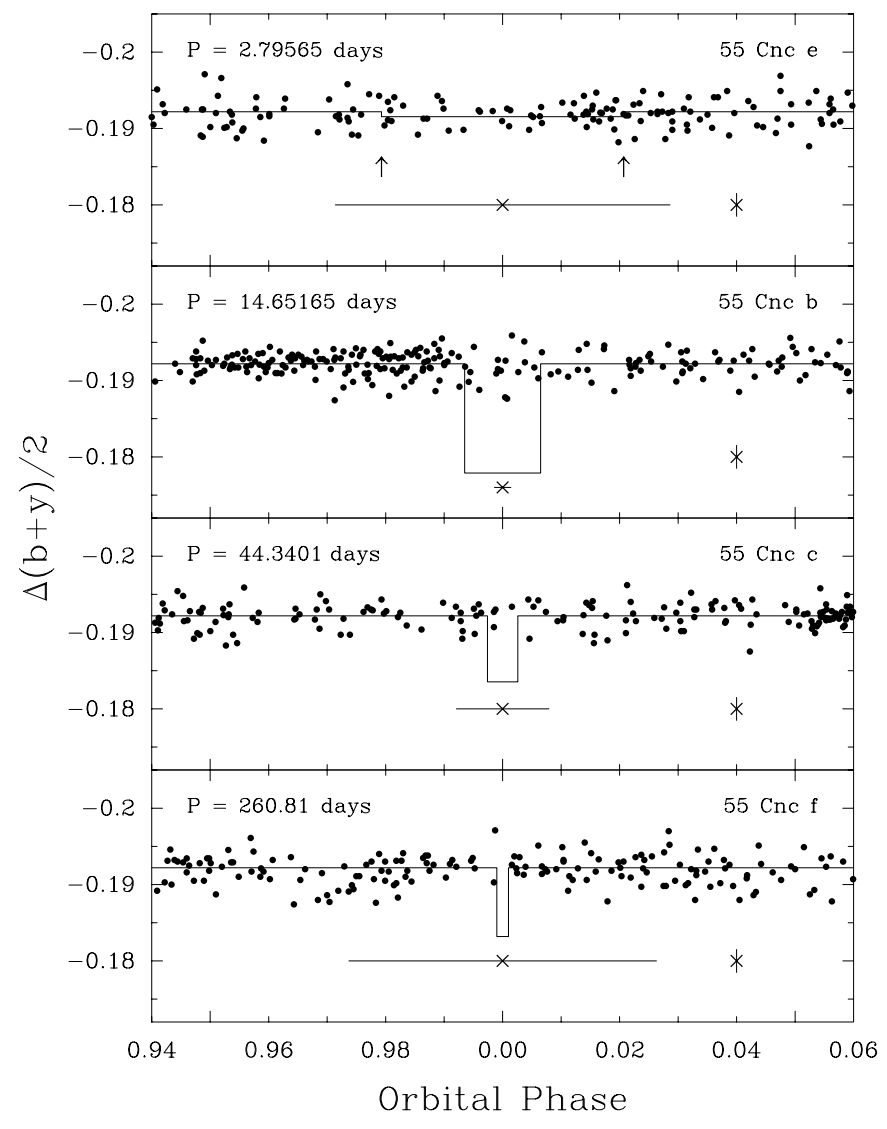

FIG. 12.-Photometric observations of $55 \mathrm{Cnc}$ plotted vs. orbital phase for the inner four planetary companions, all plotted to the same scale. The solid line in each panel approximates the predicted transit light curve, including the depth, duration, and timing of the transits. The arrows in the top panel indicate the beginning and end of the very shallow predicted transits of the inner planet. The horizontal error bar beneath each transit box indicates the uncertainty in the time of transit, while the vertical error bar shows the nominal precision of a single data point. The phase-folded photometry does not detect transits for any of the four inner planets.

radial velocity period because of the relatively large uncertainty of 2.5 days in the rotation period, the absence of any photometric variability on the more accurate 44.35 day radial velocity period is strong support for the existence of $55 \mathrm{Cnc}$.

In Figure 12, we have plotted light curves of the photometric data phased with the orbital periods of the inner four planetary companions. Zero phase in each panel represents the predicted phase of midtransit for each of the companions. Only phases from 0.94 to 0.06 are plotted to improve visibility of any possible transits. The solid curve in each panel approximates the predicted transit light curve, assuming a planetary orbital inclination of $90^{\circ}$ (central transits). The out-of-transit light level corresponds to the mean (normalized) brightness of the observations. The transit durations are calculated from the orbital elements, while the transit depths are derived from the estimated stellar radii and the planetary radii computed with the models of Bodenheimer et al. (2003). The horizontal error bar below each predicted transit curve represents the approximate $\pm 1 \sigma$ uncertainty in the time of midtransit, based on Monte Carlo simulations and the uncertainties in the orbital elements. The vertical error bar represents the typical \pm 0.0015 mag uncertainty of a single observation.

Column 4 of Table 5 lists the geometric probability of transits for each of the five companions, computed from equation (1) of Seagroves et al. (2003) and assuming random orbital inclinations. The predicted transit depths for each planet determined as de- scribed above are given in column (5), and the "observed transit depths" are recorded in column (6). The observed depths are computed as the difference in the mean light levels between observations that fall inside and outside the transit windows plotted in Figure 12; a positive depth indicates a brightness drop in the transit window.

Unfortunately, we see no evidence for transits of the inner, low-mass companion $55 \mathrm{Cnc}$ e; the mean of the 51 observations within its predicted transit window agree with the out-of-transit observations within $0.00029 \pm 0.0002 \mathrm{mag}$, a result consistent with the absence of 0.00065 mag transits but still allowing a small possibility for their existence. Since the uncertainty in the time of midtransit is rather large compared to the transit duration, we searched for shallow transits over the full range of phases and over orbital periods between 2.70 and 2.90 days with null results. The secure detection of transits of such a small body would require reducing the uncertainty of the in-transit brightness mean by a factor of about 2 , which would require a factor of 4 more observations. This could be accomplished with the APT over the next observing season or two by concentrating brightness measurements around the times of predicted transits. We are forced to leave our nondetection of transits of $55 \mathrm{Cnc}$ e as an uncertain result, as indicated by the colon in column (7) of Table 5.

Table 5 and Figure 12 demonstrate that transits with the expected depths of $55 \mathrm{Cnc} b$ and $\mathrm{c}$ do not occur. The observed transit depths are both consistent with zero. Figure 12 shows that we have no photometric observations during the predicted time of transit of $55 \mathrm{Cnc}$. However, given the uncertainty in the transit timing and the density of observations within the uncertainty range, we conclude that transits of planet $f$ probably do not occur. We have insufficient radial velocities to predict accurate transit times of the outermost planet $55 \mathrm{Cnc}$ d, so we can say nothing about their occurrence. We note that our nondetection of transits is consistent with the likely inclinations of the planetary orbits as discussed in $\S 9$ (below).

Finally, we comment on the long-term variability of the host star 55 Cnc. Although our normalization of the light curve has removed any such variation from the present analysis, an examination of the light curves computed with the secondary comparison stars mentioned above show that $55 \mathrm{Cnc}$ clearly exhibits year-to-year variations in mean brightness with an amplitude of 0.001 mag over a timescale of several years or more. Thus, longand short-term brightness variations in $55 \mathrm{Cnc}$ are very similar to irradiance variations in our Sun (e.g., Willson 1997).

\section{MINIMUM-MASS PROTOPLANETARY NEBULA}

Planet formation in the protoplanetary disk around $55 \mathrm{Cnc}$ was apparently extraordinarily efficient, yielding at least five planets. Our extensive radial velocity data set, with its $18 \mathrm{yr}$ baseline, gives no indication that additional Jupiter-mass companions exist beyond $6 \mathrm{AU}$, although Saturn-mass or smaller planets would easily go undetected. If, as a thought experiment, we grind up the currently known planets, we may infer the properties of the protoplanetary disk around $55 \mathrm{Cnc}$.

We first assume an edge-on, coplanar geometry with $i=90^{\circ}$ and in situ formation of the planets directly from the disk gas (e.g., Boss 1997). In this case, the masses of planets total $\sim 5.3 M_{\text {Jup }}$ and together imply a lower limit of $410 \mathrm{~g} \mathrm{~cm}^{-2}$ for the average surface density of the disk interior to $6 \mathrm{AU}$. For a gas-todust ratio of 100, this implies an average surface density in solids of $4 \mathrm{~g} \mathrm{~cm}^{-2}$. If the radial surface density profile of $55 \mathrm{Cnc}$ 's protostellar disk declined as $\sigma(r) \propto r^{-3 / 2}$, this implies a solid surface density of $1.4 \mathrm{~g} \mathrm{~cm}^{-2}$ at $5 \mathrm{AU}$, which is approximately half 
TABLE 5

Results of Photometric Transit Search

\begin{tabular}{|c|c|c|c|c|c|c|}
\hline $\begin{array}{c}\text { Planet }^{\mathrm{a}} \\
\text { (1) }\end{array}$ & $\begin{array}{l}\text { Planetary Period } \\
\text { (days) } \\
\text { (2) }\end{array}$ & $\begin{array}{l}\text { Semiamplitude } \\
\text { (mag) } \\
\text { (3) }\end{array}$ & $\begin{array}{c}\text { Transit Probability } \\
\text { (\%) } \\
\text { (4) }\end{array}$ & $\begin{array}{l}\text { Predicted Transit Depth } \\
\text { (mag) } \\
(5)\end{array}$ & $\begin{array}{l}\text { Observed Transit Depth } \\
\text { (mag) } \\
(6)\end{array}$ & $\begin{array}{c}\text { Transits } \\
\text { (7) }\end{array}$ \\
\hline е & 2.79565 & $0.00004 \pm 0.00006$ & 9.7 & +0.00065 & $+0.00029 \pm 0.00020$ & No? \\
\hline 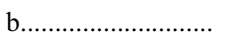 & 14.65165 & $0.00006 \pm 0.00006$ & 4.1 & +0.0143 & $+0.0007 \pm 0.0005$ & No \\
\hline с & 44.3401 & $0.00008 \pm 0.00006$ & 2.0 & +0.0086 & $-0.0003 \pm 0.0006$ & No \\
\hline 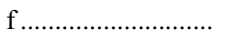 & 260.81 & $0.00008 \pm 0.00006$ & 0.8 & +0.0090 & $\mathrm{~b}$ & No: \\
\hline $\mathrm{d}$ & 5223 & $\ldots{ }^{c}$ & 0.1 & +0.0155 &.$^{\mathrm{d}}$ & $?$ \\
\hline
\end{tabular}

\footnotetext{
${ }^{a}$ Planets are listed in order of increasing orbital period; however, the planet designations, $\mathrm{b}-\mathrm{f}$, correspond to the chronological order of their discovery.

${ }^{\mathrm{b}}$ No observations in the predicted $12 \mathrm{hr}$ transit window but many observations within the $1 \sigma$ uncertainty interval.

${ }^{c}$ Duration of the photometric record is less than the planetary orbital period.

${ }^{\mathrm{d}}$ Poorly constrained orbit and insufficient photometric phase coverage.
}

the value of the minimum-mass solar nebula at Jupiter's current position.

In all likelihood, however, the surface density of solids in 55 Cnc's protostellar disk was higher than in the solar nebula. If we assume that the planets formed via the core accretion mechanism, as described, for example, by Hubickyj et al. (2005), we estimate that they contain at least $150 M_{\oplus}$ of heavy elements. Here we include the high metallicity of the host star, $55 \mathrm{Cnc}$, with its $[\mathrm{Fe} / \mathrm{H}]=+0.3$ as representative of the planet's interior. Reconstituting this mass of solids to recover 55 Cnc's metallicity implies an original protostellar disk mass of $\sim 0.025 M_{\odot}$ within $6 \mathrm{AU}$. Assuming that the nascent disk extended to $30 \mathrm{AU}$ with a $r^{-3 / 2}$ surface density profile, the total mass would have been $\sim 0.06 M_{\odot}$, and the surface mass density in solids at $5 \mathrm{AU}$ would have been $7 \mathrm{~g} \mathrm{~cm}^{-2}$.

Adopting the reported orbital inclination of $53^{\circ}$ for the outer planet (McArthur et al. 2004) and assuming the orbits to be coplanar augments all masses by $1 / \sin i=1.25$. The resulting simplistic minimum-mass protoplanetary nebula has $510 \mathrm{~g} \mathrm{~cm}^{-2}$ for the average surface mass density of the combined gas and dust within 6 AU. Adopting a nominal gas-to-dust ratio of 100 yields a dust surface mass density of $5 \mathrm{~g} \mathrm{~cm}^{-2}$.

But again considering the likely enrichment of solids within giant planets, and the associated $\mathrm{H}$ and $\mathrm{He}$, yields an original mass within $6 \mathrm{AU}$ of at least $0.031 M_{\odot}$. Extending this disk to $30 \mathrm{AU}$ gives a total mass of $0.075 M_{\odot}$. The estimated surface mass density of solids in the disk at $5 \mathrm{AU}$ would have been $8.7 \mathrm{~g} \mathrm{~cm}^{-2}$.

For expected equilibrium disk temperatures, this minimummass disk is below the threshold required for the development of nonaxisymmetric gravitational instabilities (Laughlin \& Rozyczka 1996), but likely high enough to support the formation of planets via core accretion (Robinson et al. 2006). In the context of this disk-profile scenario, the core accretion theory suggests that additional objects with masses ranging from Neptune to Saturn mass should be present beyond the frontier marked by the orbit of planet d, i.e., beyond 6 AU. If the outer planet migrated during or after its formation, the estimated disk properties computed here would be affected.

\section{DISCUSSION}

Our 18 year campaign of Doppler measurements of $55 \mathrm{Cnc}$ at the Lick Observatory and the Keck Observatory has gradually revealed additional superimposed wobbles, each best interpreted as due to another orbiting planet. The previously identified four planets revealed a large gap between 0.24 and 5.8 AU, raising questions about unseen planets there and the planet formation history in the protoplanetary disk. The velocities presented here reveal a fifth periodicity with $P=260$ days, consistent with Keplerian motion, for which the most reasonable interpretation is another orbiting planet. The five-planet model suggests the new planet has a minimum mass of $45 M_{\oplus}$ in a nearly circular orbit with a semimajor axis of $a=0.781 \mathrm{AU}$. Thus, $55 \mathrm{Cnc}$ is the first quintuple-planet system known.

This fifth planet apparently resides in the previously identified gap between 0.24 and $5.8 \mathrm{AU}$, and it remains between $0.73 \mathrm{AU}$ (periastron) and $0.84 \mathrm{AU}$ (apastron), preventing orbit crossings with both the next inner planet, c, whose apastron is at $0.26 \mathrm{AU}$, and the outer planet, $\mathrm{d}$, whose periastron is at 5.5 AU, ensuring dynamical stability that is demonstrated numerically by $N$-body simulations. As the star's luminosity is $L=0.60 L_{\odot}$ (from its effective temperature and radius), this fifth planet resides within the classical habitable zone. Given its minimum mass of $45 M_{\oplus}$, we speculate that it contains a substantial amount of hydrogen and helium, not unlike Saturn $\left(M=95 M_{\oplus}\right)$ in the solar system.

The four previously published planets around $55 \mathrm{Cnc}$ now have revised orbital parameters and masses because the fifth planet had been contaminating the Doppler signal but was not taken into account. The orbital semimajor axes and masses of all five planets (moving outward from the central star) are $a=0.038 \mathrm{AU}$ and $M \sin i=10.8 M_{\oplus}, 0.115 \mathrm{AU}$ and $0.824 M_{\mathrm{Jup}}, 0.24 \mathrm{AU}$ and $0.169 M_{\text {Jup }}, 0.781 \mathrm{AU}$ and $0.144 M_{\text {Jup }}$, and 5.77 AU and 3.83 $M_{\text {Jup }}$. All quoted minimum masses are uncertain by $\sim 5 \%$ due to the uncertain mass of the host star $55 \mathrm{Cnc}$. The planets in this system all have nearly circular orbits, with the caveat that the orbital eccentricity for the 260 day planet is poorly constrained by radial velocity data.

The inclination of the orbital plane of the outer planet has been estimated from the apparent astrometric motion of the star, as measured with the Fine Guidance Sensor on the Hubble Space Telescope (McArthur et al. 2004). The derived orbital inclination is $i=53^{\circ} \pm 6.8^{\circ}$ ( $37^{\circ}$ from edge-on) for that outer planet, implying that its actual mass is $4.9 M_{\text {Jup }}$. Assuming coplanarity for the other four planets, their actual masses (proceeding outward) are $M_{e}=13.5 M_{\oplus}$ (nearly one Uranus mass), $M_{b}=1.03 M_{\mathrm{Jup}}$, $M_{c}=0.21 M_{\text {Jup }}=66.7 M_{\oplus}$, and $M_{f}=0.18 M_{\text {Jup }}=57 M_{\oplus}$. Normally, coplanarity should not be a foregone conclusion, as the eccentricities of many exoplanets imply a dynamically perturbative history. But for the $55 \mathrm{Cnc}$ system with its five planets so vulnerable to instabilities, such an active past seems unlikely. Any great perturbations would have ejected the smaller planets. Thus, the planetary orbits in $55 \mathrm{Cnc}$ are likely to reside in a flattened plane, analogous to the ecliptic, coinciding with the original protoplanetary disk out of which the planets presumably formed. 
If 55 Cnc did have a quiescent past, the star's spin axis should be nearly coincident with the normal of the system's orbital plane. The inclination of the spin axis can be determined from the spectroscopically measured rotational $v \sin i=2.46 \mathrm{~km} \mathrm{~s}^{-1}$ and the photometrically determined spin period of 42.7 days for the star, along with its radius of $0.93 R_{\odot}$. The 42.7 day spin period implies an equatorial velocity of only $1.24 \mathrm{~km} \mathrm{~s}^{-1}$, which is lower than $v \sin i$, an impossibility. Either the measured $v \sin i$ is too high by a factor of 2 (quite possible given the many line-broadening sources) or the spin period is much shorter (not likely, given the low chromospheric activity). As is common, the inclination of the spin axis of a nearby star, as attempted here, carries uncertainties so large as to be of little value. Nonetheless, the crude interpretation would be that the star is not viewed pole-on. Indeed, if the orbits were viewed nearly pole-on the implied planetary masses would be so large as to render the system dynamically unstable. We conclude that the orbital plane of $55 \mathrm{Cnc}$ is not being viewed nearly pole-on, consistent with the astrometric value of $i=53^{\circ}$.

As the $55 \mathrm{Cnc}$ system has more planets than any previously discovered system, its overall structure, including its planet mass distribution, its density of orbits, and its orbital eccentricities, offers direct constraints on its protoplanetary disk and subsequent planetary dynamics. The 55 Cnc system may initially be sketched as having one massive planet, likely a hydrogen-helium gas giant, in a nearly circular orbit at 5.8 AU. Inward are four less massive planets, the innermost being roughly Uranus mass, the next outward having roughly Jupiter mass and likely gaseous, and the next two having somewhat sub-Saturn masses, also probably gaseous. The outer planet has an orbital angular momentum quickly shown to be at least $8.2 \times 10^{43} \mathrm{~kg} \mathrm{~m}^{2} \mathrm{~s}^{-1}$, certainly 100 times greater than the star's spin angular momentum, $6.1 \times 10^{41} \mathrm{~kg} \mathrm{~m}^{2} \mathrm{~s}^{-1}$ (from its 42.7 day spin period).

Thus, 55 Cnc contains a dominant outer planet at $5.8 \mathrm{AU}$ of roughly $5 M_{\text {Jup }}$ and four lower mass planets, all five in nearly circular orbits (although two orbits appear to be somewhat more eccentric than found for the more massive planets in our solar system). The large eccentricities found in the majority of exoplanets (Marcy et al. 2005; Butler et al. 2006) are not seen in $55 \mathrm{Cnc}$. The five orbits in $55 \mathrm{Cnc}$ are probably nearly coplanar, as discussed above, lest some planet masses be too large to allow stability. Thus, the $55 \mathrm{Cnc}$ system has some basic structural attributes found in our solar system: nearly coplanar, circular orbits, with a dominant gas giant between 5 and 6 AU. This similarity suggests that such solar system architectures are not extremely rare.

The formation of multiplanet systems with outer, dominant planets may occasionally form such that they persist for billions of years without disruptive gravitational perturbations that cause large eccentricities and eject planets. Because nested, coplanar, circular orbits could hardly be obtained unless they began that way, the 55 Cnc system, along with the solar system, supports the hypothesis that planets form in viscous protoplanetary disks, as has long been predicted by standard planet formation theory (e.g., Lissauer 1995).

One puzzle is whether the $55 \mathrm{Cnc}$ planets suffered significant migration. The 44.35 and 14.65 day planets have a period ratio of $3.027: 1.000$, thus leaving open the possibility of a mean motion resonance identified previously (Marcy et al. 2002). As planets have no reason to form with integer period ratios, any resonance suggests that some differential migration occurred, allowing the two planets to capture each other. However, the $3: 1$ mean motion resonance was found to be absent in the current $N$-body model as none of the relevant resonant arguments are in libration.
However, if migration occurred, we wonder what prevented the outer planet, and indeed all of the planets, from migrating inward. Perhaps the disk dissipated just as this last crop of five planets formed, as suggested in some migrational models (Lin et al. 2000; Trilling et al. 2002; Armitage et al. 2002, 2003; Ida \& Lin 2004, 2005; Narayan et al. 2005). Indeed, the proximity of the three inner planets to the host star, especially the Jupiter-mass planet at $0.115 \mathrm{AU}(P=14.65$ days $)$, suggests that they migrated inward to their present locations, assuming they did not form in situ. If so, protoplanetary disk material likely orbited outside $0.24 \mathrm{AU}$, exerting an inward torque on those planets and carrying away orbital angular momentum in the system. During the migration period, the implied disk material would have had a mass comparable to (or exceeding) the Jovian-mass planets, from which the most recently identified planet at 0.78 AU could have formed.

It is interesting that the third and fourth planets (at 0.24 and $0.78 \mathrm{AU}$ ) have small minimum masses, under $0.2 M_{\text {Jup }}$, but are surrounded by much larger giant planets with minimum masses of 0.824 and $3.8 M_{\text {Jup }}$. One wonders why the acquisition of material was apparently so different among these four planets.

One also wonders why this particular star ended up with five planets while $90 \%$ of stars on Doppler surveys do not have any detected giant planets. Perhaps the high metallicity of $55 \mathrm{Cnc}$ $([\mathrm{Fe} / \mathrm{H}]=+0.30)$ played a role in the efficient planet formation. Fischer \& Valenti (2005) find a correlation not only between stellar metallicity and the occurrence of planets, but also between high metallicity and multiplanet systems. But we doubt that a mere factor of 2 enhancement in heavy elements could account entirely for the five planets in this system. Some stochasticity in planet formation and subsequent stability must play a role.

The outer planet at 5.8 AU is angularly separated from the star $(d=12.5 \mathrm{pc})$ by $0.47^{\prime \prime}$, making it a good target for nextgeneration adaptive optics systems. The Space Interferometry Mission PlanetQuest (SIM PlanetQuest), operating in narrowangle mode with astrometric precision of $1 \mu \mathrm{as}$, could measure the astrometric wobble caused by all four outer planets, providing definitive masses and orbital inclinations for them. A space-borne coronagraph or a space-borne interferometer might be capable of imaging the outer planet and taking its spectra. NASA and ESA have a wonderful opportunity to fund such an imaging telescope, thereby detecting and spectroscopically assessing a mature extrasolar planet. Moreover, as $55 \mathrm{Cnc}$ is metal-rich, the planets may also be abundant in heavy elements, offering an opportunity to study rich atmospheric chemistry, clouds, and weather, if spectra could be obtained. Transits, if any occur, would provide planet radii offering information about potential rocky cores.

This rich planetary system portends a fruitful future for the Doppler technique of studying exoplanets. It shows that extending the time baseline of Doppler measurements can reveal multiple planets, the existence or absence of which provides information about the formation, structure, and evolution of planetary systems.

We gratefully acknowledge the efforts and dedication of the Lick and Keck Observatory staff. We thank Karl Stapelfeldt for helpful comments. We thank the anonymous referee for comments that improved the manuscript. We appreciate support by NASA grant NAG5-75005 and by NSF grant AST 03-07493 to S. S. V.; and support by NSF grant AST 99-88087, by NASA grant NAG512182, and travel support from the Carnegie Institution of Washington to R. P. B. G. W. H. acknowledges support from 
NASA grant NCC5-511 and NSF grant HRD-9706268. We are also grateful for support by Sun Microsystems. We thank the NASA and UC Telescope assignment committees for allocations of telescope time toward the planet search around M dwarfs. This research has made use of the SIMBAD database, operated at CDS, Strasbourg, France. The authors wish to extend special thanks to those of Hawaiian ancestry on whose sacred mountain of Mauna Kea we are privileged to be guests. Without their generous hospitality, the Keck observations presented herein would not have been possible.
Adams, F. C., \& Laughlin, G. 2006, ApJ, 649, 1004

Armitage, P. J., Clarke, C. J., \& Palla, F. 2003, MNRAS, 342, 1139

Armitage, P. J., Livio, M., Lubow, S. H., \& Pringle, J. E. 2002, MNRAS, 334, 248

Baliunas, S. L., Donahue, R. A., Soon, W., \& Henry, G. W. 1998, in ASP Conf. Ser. 154, Cool Stars, Stellar Systems, and the Sun, ed. R. A. Donahue \& J. A. Bookbinder (San Francisco: ASP), 153

Bodenheimer, P., Laughlin, G., \& Lin, D. N. C. 2003, ApJ, 592, 555

Boss, A. P. 1997, Science, 276, 1836

Bryden, G., et al. 2006, ApJ, 636, 1098

Butler, R. P., Marcy, G. W., Fischer, D. A., Brown, T. M., Contos, A. R., Korzennik, S. G., Nisenson, P., \& Noyes, R. W. 1999, ApJ, 526, 916

Butler, R. P., Marcy, G. W., Williams, E., Hauser, H., \& Shirts, P. 1997, ApJ, 474, L115

Butler, R. P., Marcy, G. W., Williams, E., McCarthy, C., Dosanjh, P., \& Vogt, S. S. 1996, PASP, 108, 500

Butler, R. P., Vogt, S. S., Marcy, G. W., Fischer, D. A., Wright, J. T., Henry, G. W., Laughlin, G., \& Lissauer, J. J. 2004, ApJ, 617, 580

Butler, R. P., et al. 2006, ApJ, 646, 505

Eggenberger, A., Udry, S., \& Mayor, M. 2004, A\&A, 417, 353

Fischer, D. A., \& Valenti, J. A. 2005, ApJ, 622, 1102

Hall, J. C., Henry, G. W., \& Lockwood, G. W. 2007, AJ, 133, 2206

Henry, G. W. 1999, PASP, 111, 845

Henry, G. W., Baliunas, S. L., Donahue, R. A., Fekel, F. C., \& Soon, W. 2000, ApJ, 531, 415

Hubickyj, O., Bodenheimer, P., \& Lissauer, J. J. 2005, Icarus, 179, 415

Ida, S., \& Lin, D. N. C. 2004, ApJ, 604, 388 . 2005, ApJ, 626, 1045

Kjeldsen, H., et al. 2005, ApJ, 635, 1281

Laughlin, G., \& Rozyczka, M. 1996, ApJ, 456, 279

Lin, D. N. C., Papaloizou, J. C. B., Terquem, C., Bryden, G., \& Ida, S. 2000, in Protostars and Planets IV, ed. V. Mannings \& A. P. Boss (Tucson: Univ. Arizona Press), 1111

Lissauer, J. J. 1995, Icarus, 114, 217

Maness, H. L., Marcy, G. W., Ford, E. B., Hauschildt, P. H., Shreve, A. T., Basri, G. B., Butler, R. P., \& Vogt, S. S. 2007, PASP, 119, 90

Marcy, G., Butler, R. P., Fischer, D., Vogt, S., Wright, J. T., Tinney, C. G., \& Jones, H. R. A. 2005, Prog. Theor. Phys. Suppl., 158, 24

Marcy, G. W., Butler, R. P., Fischer, D. A., Laughlin, G., Vogt, S. S., Henry, G. W., \& Pourbaix, D. 2002, ApJ, 581, 1375

\section{REFERENCES}

McArthur, B. E., et al. 2004, ApJ, 614, L81

Murray, C. D., \& Dermott, S. F. 1999, Solar System Dynamics (Cambridge: Cambridge Univ. Press)

Narayan, R., Cumming, A., \& Lin, D. N. C. 2005, ApJ, 620, 1002

Noyes, R. W., Hartmann, L. W., Baliunas, S. L., Duncan, D. K., \& Vaughan, A. H. 1984, ApJ, 279, 763

Paulson, D. B., Saar, S. H., Cochran, W. D., \& Henry, G. W. 2004, AJ, 127, 1644

Press, W. H., Teukolsky, S. A., Vetterling, W. T., \& Flannery, B. P. 1992, Numerical Recipes in FORTRAN (2nd ed.; Cambridge: Cambridge Univ. Press)

Queloz, D., et al. 2001, A\&A, 379, 279

Raghavan, D., Henry, T. J., Mason, B. D., Subasavage, J. P., Jao, W.-C., Beaulieu, T. D., \& Hambly, N. C. 2006, ApJ, 646, 523

Raymond, S. N., Barnes, R., \& Kaib, N. A. 2006, ApJ, 644, 1223

Richardson, L. J., Deming, D., Horning, K., Seager, S., \& Harrington, J. 2007, Nature, 445, 892

Rivera, E. J., et al. 2005, ApJ, 634, 625

Robinson, S. E., Laughlin, G., Bodenheimer, P., \& Fischer, D. 2006, ApJ, 643, 484

Santos, N. C., et al. 2004, A\&A, 426, L19

Sato, B., et al. 2005, ApJ, 633, 465

Schneider, G., Becklin, E. E., Smith, B. A., Weinberger, A. J., Silverstone, M., \& Hines, D. C. 2001, AJ, 121,525

Seagroves, S., Harker, J., Laughlin, G., Lacy, J., \& Castellano, T. 2003, PASP, 115,1355

Takeda, G., Ford, E. B., Sills, A., Rasio, F. A., Fischer, D. A., \& Valenti, J. A. 2007, ApJS, 168, 297

Trilling, D. E., Lunine, J. I., \& Benz, W. 2002, A\&A, 394, 241

Valenti, J. A., \& Fischer, D. A. 2005, ApJS, 159, 141

VandenBerg, D. A., \& Clem, J. L. 2003, AJ, 126, 778

Vaníček, P. 1971, Ap\&SS, 12, 10

Vogt, S. S. 1987, PASP, 99, 1214

Vogt, S. S., et al. 1994, Proc. SPIE, 2198, 362

Willson, R. C. 1997, Science, 277, 1963

Wisdom, J. 2005, BAAS, 37, 525

Wright, J. T. 2005, PASP, 117, 657

Wright, J. T., Marcy, G. W., Butler, R. P., \& Vogt, S. S. 2004, ApJS, 152, 261

Yi, S. K., Demarque, P., \& Kim, Y.-C. 2004, Ap\&SS, 291, 261 